\title{
On Emergent Particles and Stable Neutral Plasma Balls in SU(2) Yang-Mills Thermodynamics
}

\author{
Ralf Hofmann ${ }^{1, *}$ and Thierry Grandou ${ }^{2}$ \\ 1 Institut für Theoretische Physik, Universität Heidelberg, Philosophenweg 12, D-69120 Heidelberg, Germany \\ 2 Institut de Physique de Nice, Université Cote d'Azur, UMR CNS 7010, 1361 Routes des Lucioles, \\ 06560 Valbonne, France; thierry.grandou@inphyni.cnrs.fr \\ * Correspondence: r.hofmann@thphys.uni-heidelberg.de
}

Citation: Hofmann, R.; Grandou, T. On Emergent Particles and Stable

Neutral Plasma Balls in SU(2)

Yang-Mills Thermodynamics.

Universe 2022, 8, 117. https:/ /

doi.org/10.3390/universe 8020117

Academic Editor: Douglas Singleton

Received: 4 January 2022

Accepted: 10 February 2022

Published: 12 February 2022

Publisher's Note: MDPI stays neutral with regard to jurisdictional claims in published maps and institutional affiliations.

Copyright: (C) 2022 by the authors. Licensee MDPI, Basel, Switzerland. This article is an open access article distributed under the terms and conditions of the Creative Commons Attribution (CC BY) license (https:// creativecommons.org/licenses/by/ $4.0 /)$.

\begin{abstract}
For a pure SU(2) Yang-Mills theory in 4D, we revisit the spatial (3D), ball-like region of radius $r_{0}$ in its bulk subject to the pressureless, deconfining phase at $T_{0}=1.32 T_{\mathcal{C}}$, where $T_{\mathcal{C}}$ denotes the critical temperature for the onset of the deconfining-preconfining phase transition. Such a region possesses finite energy density and represents the self-intersection of a figure-eight shaped centervortex loop if a BPS monopole of core radius $\sim \frac{r_{0}}{52.4}$, isolated from its antimonopole by repulsion externally invoked through a transient shift of (anti)caloron holonomy (pair creation), is trapped therein. The entire soliton (vortex line plus region of self-intersection of mass $m_{0}$ containing the monopole) can be considered an excitation of the pressureless and energyless ground state of the confining phase. Correcting an earlier estimate of $r_{0}$, we show that the vortex-loop self-intersection region associates to the central part of a(n) (anti)caloron and that this region carries one unit of electric $\mathrm{U}(1)$ charge via the (electric-magnetic dually interpreted) charge of the monopole. The monopole core quantum vibrates at a thermodynamically determined frequency $\omega_{0}$ and is unresolved. For a deconfining-phase plasma oscillation about the zero-pressure background at $T=T_{0}$, we compute the lowest frequency $\Omega_{0}$ within a neutral and homogeneous spatial ball (no trapped monopole) in dependence of its radius $R_{0}$. For $R_{0}=r_{0}$ a comparison of $\Omega_{0}$ with $\omega_{0}$ reveals that the neutral plasma oscillates much slower than the same plasma driven by the oscillation of a monopole core.
\end{abstract}

Keywords: SU(2) Yang-Mills thermodynamics; deconfining phase; caloron; confining phase; centervortex loop; electron; BPS monopole; quantum of action

\section{Introduction}

The thermodynamical phase structure of a single SU(2) Yang-Mills theory (electricmagnetic dually interpreted with respect to $\mathrm{U}(1) \subset \mathrm{SU}(2))$, comprising deconfining and preconfining thermal ground states of finite energy densities and (partially) massive gaugefield excitations as well as an energyless and pressureless confining ground state, suggests the existence of a solitonic, stable particle with intriguing yet familiar properties. Immersed into the confining ground state [1], the figure-eight configuration of a self-intersecting centervortex loop acquires its two-fold degenerate magnetic moment by a quantised electric current, composed of a chain of alternating monopoles and antimonopoles [2]. The mass $m_{0}$ of this soliton mainly arises from deconfining energy density within the self-intersection region of the vortex loop, idealised to be a spherical blob of radius $r_{0}$. At a temperature $T_{0}=1.32 T_{\mathcal{C}}$, where $T_{\mathcal{C}}$ is the boundary ${ }^{1}$ of the deconfining phase, the pressure vanishes with positive slope (stable particle) [1]. The electric charge of this region is carried by a trapped BPS monopole of mass $m_{m} \ll m_{0}$ which is not resolved thermodynamically and orginated by large-holonomy (anti)caloron dissociation (pair creation) [3]. The monopole's core fluctuates and is quantum initiated [1] to breathe at a certain frequency $\omega_{0}[4,5]$.

It is tempting to interpret this quantum soliton as the electron (or an idealised charged lepton void of weak decay) (see, however, [6] for an interesting, extended field configuration 
mapping Minkowski to the internal space $S_{3}$ and argued to describe the electron's quantum numbers on the classical level in terms of topological charges). In the present paper (see also [7]), we realise Louis de Broglie's ideas on the (quantum) thermodynamics of the isolated particle in its rest frame [8,9] subject to internal, spatially homogeneous 'clock ticks' at rate $\omega_{0}$. According to de Broglie, such a spatially localised (within a 3D ball-like volume), time-periodic phenomenon prescribes the soliton mass $m_{0}$ via $m_{0} c^{2}=\hbar \omega_{0}$. By Lorentzboosting the standing wave of the particle at rest to a propagating wave (particle at spatial momentum $p$ ), this implies the well-known matter wavelength $\lambda=\frac{2 \pi \hbar}{p}$ — the starting point for the development of wave mechanics [10-13]. The probabilistic interpretation of the locus of electric charge in terms of the wavefunction's squared amplitude [14] matches with the fact that the periodically breathing, unresolved monopole core is displaced frequently and undeterministically by a local engagement with $\hbar$ (and an external field, e.g., in a hydrogen atom) throughout the self-intersection region of the vortex. The free solitons's apparent structurelessness, as inferred from collider experiments, is due to the thermal nature of the self-intersection region (maximum entropy): Electric monopole charges equally likely occur at any spatial point within volume $\sim \frac{4}{3} \pi r_{0}^{3}$, which is, in turn, immersed into the ground state of the confining phase-a condensate of shrunk-to-points centervortex loops void of pressure and energy density [1]. (On the scale of rest mass $m_{0}$, the nonself-intersecting, spatial center-vortex loop is massless and prone to curve shrinking $[1,15]$.)

In the present paper, we discuss a few amendments to [7], where the above-sketched model for a charged lepton (idealised to be stable against weak decay) was introduced, and we address oscillation physics about temperature $T_{0}$ of a neutral plasma ball to conclude that the contributions to the quantum mass of the soliton from low-lying frequencies of such spherically symmetric breathing modes are negligible. In Section 2, we correct the values of $r_{0}$ and the monopole mass $m_{m}$ as well as the ratio $m_{m} / m_{0}$ based on the monopole mass formula:

$$
m_{m}=\frac{4 \pi}{e\left(T_{0}\right)} H_{\infty}\left(T_{0}\right)
$$

where $H_{\infty}\left(T_{0}\right)=\pi T_{0}[1,3,16-22]$, and $e\left(T_{0}\right)=12.96$ is the value of the effective gauge coupling at $T_{0}[1]$. In writing Equation (1), we assume that the spatial asymptotics of the monopole's $A_{4}$ (or adjoint Higgs) field is determined by temperature alone, reflecting the fact that the maximum holonomy of the originating, dissociated (anti)caloron is enforced externally and not influenced by the ensemble's spatial finite-range correlations encoded in the value of $e\left(T_{0}\right)=12.96 \neq 1$. The ratio $\frac{r_{0}}{|\phi|^{-1}\left(T_{0}\right)}$ is substantially smaller than erroneously estimated in [7]: Instead of $\frac{r_{0}}{|\phi|^{-1}\left(T_{0}\right)} \sim 160$, we now have $\frac{r_{0}}{|\phi|^{-1}\left(T_{0}\right)} \sim 0.1033$. Here, $|\phi|(T)=$ $\sqrt{\frac{\Lambda^{3}}{2 \pi T}}$ represents the modulus of the adjoint and inert scalar field of the deconfining phase (spatially coarse-grained, densely packed (anti)caloron centers [1]), and $\Lambda$ denotes the Yang-Mills scale. The length scale $|\phi|^{-1}$, therefore, sets the resolution for the SU(2) gauge-field theory prescribed by the quantum behavior of (anti)caloron centers [1]. In the present paper, we show that $\frac{r_{0}}{T_{0}^{-1}} \sim 1.29$. Therefore, the blob of center-vortex selfintersection $^{2}$ does not per se represent infinite-volume thermodynamics [1]. Rather, it is deeply contained within the central (quantum) region of the accomodating caloron or anticaloron: $\frac{r_{0}}{|\phi|^{-1}\left(T_{0}\right)} \sim 0.1033$. We will argue, however, that as far as the derivation of soliton properties is concerned, the thermodynamical results in [1] still apply. Interestingly, the smallness of $\frac{r_{0}}{|\phi|^{-1}\left(T_{0}\right)}$ excludes the possibility of trapping two or more monopoles or antimonopoles within one and the same blob: they would have to be provided by two or more dissociating (anti)calorons but the blob only contains the central part of a single (anti)caloron. In addition to a re-visit of the physics of the self-intersection region at temperature $T_{0}$ in a spatial center-vortex loop, we also discuss, in Section 3 , the lowest radial oscillation of a neutral deconfining plasma ball of radius $R_{0}$ which does not trap a monopole. Computing the associated frequency $\Omega_{0}$ requires the determination of longitudinal sound speed $c_{S}$ at $T_{0}$. We obtain $c_{S}=0.479 c$. Note that in spite of $T_{0} \sim T_{c}$, where conformal- 
symmetry breaking effects due to the Yang-Mills scale $\Lambda$ are large, this value is close to the ultra-relativistic-gas limit $c_{S}=\frac{1}{\sqrt{3}} c \sim 0.577 c$. To exclude that there are sizable corrections to the left-hand side of Equation (5) due to plasma-breathing effects just above the threshold we compare $\Omega_{0}$ and $\omega_{0}=m_{0} c^{2} / \hbar$ at $R_{0}=r_{0}$ and find $\Omega_{0} \ll \omega_{0}$. Finally, in Section 4 , we summarise our work and comment on future developments.

\section{Self-Intersection Region of a Figure-Eight Shaped Center-Vortex Loop}

In [7], a model of the free electron was proposed which relies on the phase structure and thermodynamical quantities of SU(2) Yang-Mills thermodynamics, the nature of the excitations in the confining phase [23], and the work in [4,5] on the perturbed BPS monopole. In [23], an operator - the 't Hooft loop—was defined nonlocally for pure SU(N) Yang-Mills theory. The 't Hooft loop is dual in nature relative to the (spatial) Wilson loop. Its action creates one unit of magnetic flux with respect to the maximal Abelian subgroub $\mathrm{U}(1)^{\mathrm{N}-1} \subset \mathrm{SU}(\mathrm{N})$, as expressed by a phase change through a root of unity in the Wilson loop linking to it. In the confining phase of $\mathrm{SU}(2)$, such a flux line occurs as the zero-coresize limit of the Abrikosov-Nielsen-Olesen vortex of winding number unity and, thus, is massless, causing 't Hooft loop to acquire a finite, spatially homogeneous expectation. Moreover, no explicit isolated charges with respect to $\mathrm{U}(1) \subset \mathrm{SU}(2)^{3}$, which could serve as flux sources or sinks, are tolerated by the pressureless confining ground state, composed of shrunk-to-points single center-vortex loops [1]. Therefore, a given, static vortex line, viewed as a $1 \mathrm{D}$ object in $3 \mathrm{D}$ space, has to form a closed loop. However, the self-intersection in a center-vortex loop locally represents a strong distortion of confining order (the 't Hooft loop representing the order parameter), reinstating a region of pressureless, deconfining phase wherein an isolated charge-a BPS monopole or antimonopole-may lead a shielded longtime existence. The responses of such a classical BPS (anti)monopole to a spherically symmetric initial perturbation and the spectrum of normal modes were investigated in $[4,5]$, respectively. As a result, it was found that the asymptotic state of oscillation is determined by a frequency $\omega_{0}$ given by the mass of the two off-Cartan modes in the adjoint Higgs model that the monopole lives in. (In a thermal situation, this adjoint Higgs model is the pure Yang-Mills theory with the $A_{4}$-component of the gauge field, for which its value at spatial infinity determines the nontrivial (anti)caloron holonomy, playing the role of the Higgs field for the spatial components $A_{i}$ [20-22].)

We now revise the implications of the incorrect monopole mass formula in [7] (Equation (18) of [7]). Moreover, we point out an error in Equation (21) of [7] which requires a conceptual reinterpretation of the physics of the self-intersection region. The mass $m_{m}$ of a BPS monopole is defined as the spatial integral of the 00-component of the energy-momentum tensor on its field configuration with winding number one in $\Pi_{2}\left(\mathrm{SU}(2) \backslash \mathrm{U}(1)=S_{2}\right)$ [24]. It reads as follows.

$$
m_{m}=\frac{4 \pi}{e} H_{\infty}
$$

Here, $e$ denotes the defining gauge coupling of the adjoint Higgs model (or the fundamental, thermalised pure $\mathrm{SU}(2)$ Yang-Mills theory), and $H_{\infty}$ is the spatially asymptotic modulus of the Higgs field. Only (anti)calorons of scale parameter close to $|\phi|^{-1}$ contribute to the emergence of the thermal ground state in the deconfining phase. Therefore, the gauge coupling $e$ can be interpreted as the effective one [1].

Assuming that the monopole was liberated by the dissociation of a maximum-holonomy caloron at $T_{0}=1.32 T_{\mathcal{c}}$, we have the following [1,3].

$$
e\left(T_{0}\right)=12.96, \quad H_{\infty}\left(T_{0}\right)=\pi T_{0} .
$$

In writing the maximum nontrivial holonomy value of $H_{\infty}\left(T_{0}\right)$, we assume that this (externally imposed, pair creation) spatial asymptote solely depends on temperature and is not influenced by the spatial finite-range correlations imposed by the trivial-holonomy (anti)caloron constituting the thermal ground-state estimate. Being a quantum soliton 
of circular frequency $\omega_{0}$, the electron's rest mass $m_{0}$ is determined by a monopole-core oscillation $[8,9]$. This frequency $\omega_{0}$ was found to be equal to the mass of the two off-Cartan modes in the adjoint Higgs model containing the BPS monopole [4,5].

$$
\omega_{0}=e H_{\infty}
$$

Alternatively, assuming a conserved energy content of the approximately ball-like self-intersection region of radius $r_{0}$ (the contribution of the two vortex loops is negligible [1], and $r_{0}$ is denoted as $R_{0}$ in [7])—trapping a quantum non-initialised, static BPS monopole of mass $m_{m}$ and constituted by a deconfining SU(2) Yang-Mills plasma of energy density $\rho\left(T_{0}\right)$ - one derives the following equation [7].

$$
\begin{aligned}
m_{0} & =\omega_{0}=12.96 H_{\infty}\left(T_{0}\right)=m_{m}+\frac{4 \pi}{3} r_{0}^{3} \rho\left(T_{0}\right) \\
& =H_{\infty}\left(T_{0}\right)\left(\frac{4 \pi}{12.96}+8.31 \times \frac{128 \pi}{3}\left(\frac{r_{0}}{18.31}\right)^{3} H_{\infty}^{3}\left(T_{0}\right)\right) .
\end{aligned}
$$

Note that the left-hand side describes the soliton mass by a situation of an initialised monopole and its covibrating quantum surroundings-all captured by a multiplication of the frequency $\omega_{0}$ with the quantum of action $\hbar$ after the oscillation was triggered by monopole interaction with the thermal ground state and its excitations-while the righthand side describes the energy balance of the system before such an initialisation has occurred. Energy conservation implies the equality of these two expressions. Notice that $m_{0}=12.96 H_{\infty}\left(T_{0}\right)$ entails that the Yang-Mills scale $\Lambda$ relates to $m_{0}$ as follows [7].

$$
\Lambda=\frac{1}{118.6} m_{0}
$$

Solving Equation (5) for $r_{0}$ yields the following:

$$
r_{0}=4.043 H_{\infty}^{-1}
$$

instead of $r_{0}=4.10 \mathrm{H}_{\infty}^{-1}$, as obtained in [7]. It is instructive to compute the relative contribution of $m_{m}$ to $m_{0}$, the ratio of the monopole core size $r_{c}$ to $r_{0}$, and the reduced Compton wave length $l_{C}$ to the thermodynamical resolution scale $|\phi|^{-1}\left(T_{0}\right)$. Note that $l_{C}=r_{c}=m_{0}^{-1}=\frac{1}{12.96 H_{\infty}\left(T_{0}\right)}[4,5]$. One has the following.

$$
\frac{m_{m}}{m_{0}}=\frac{4 \pi}{(12.96)^{2}}=0.0748, \frac{r_{c}}{r_{0}}=\frac{1}{52.40}, \frac{l_{C}}{|\phi|^{-1}\left(T_{0}\right)}=0.00197 .
$$

Therefore, a hypothetically static, classical monopole would not contribute sizably to the mass of the region of self-intersection. Rather, it is the phenomenon of oscillation, expressing a strong interdependence between the unresolved monopole and its quantum environment that gives rise to the mass of this region. The region's radius $r_{0}$ is about 50-times larger than the radius of the monopole core, and the monopole core intrinsically is far from being resolved. Therefore, it is safe to say that the quantum-induced motion of the monopole is not influenced by the boundary. This boundary is the surface where the plasma of the self-intersection region transmutes into a thin fuzzy/turbulent shell of preconfining phase adjacent to a surrounding of pressureless confining ground state, which is composed of condensed single and shrunk-to-points center vortices [1]. Interestingly, radius $r_{0}$ compares to the spatial coarse-graining scale $|\phi|^{-1}\left(T_{0}\right)$ as follows.

$$
\frac{r_{0}}{|\phi|^{-1}\left(T_{0}\right)}=\frac{4.043}{\sqrt{2\left(\frac{118.6}{12.96}\right)^{3}}}=0.1033 \text {. }
$$


A simple calculational error in Equation (22) of [7] has produced a value much larger than unity. The correct result of Equation (9) implies that the "thermodynamics" we discussed so far actually occurs deep within the center of a(n) (anti)caloron of scale parameter $\rho \sim|\phi|^{-1}\left(T_{0}\right)$. Since we have the following:

$$
\frac{r_{0}}{\beta_{0}}=1.29 \quad\left(\beta_{0} \equiv \frac{1}{T_{0}}\right) \text {, }
$$

Figure 1 suggests that the scale parameter integral, defining the phase of $\phi[1]$, does not quite saturating a harmonic dependence on Euclidean time when cut off at $\rho \sim r_{0}$. However, our above discussion on the right-hand side of Equation (5) assumes that such a saturation occurs within the volume $\frac{4 \pi}{3} r_{0}^{3}$. Hence, the right-hand side of Equation (5) only yields an approximate account of the distorted thermodynamics within the self-intersection region: the monopole is always close to the locus of action at the inmost point of the caloron or anticaloron, rendering this region a jittery object even within its deep bulk.
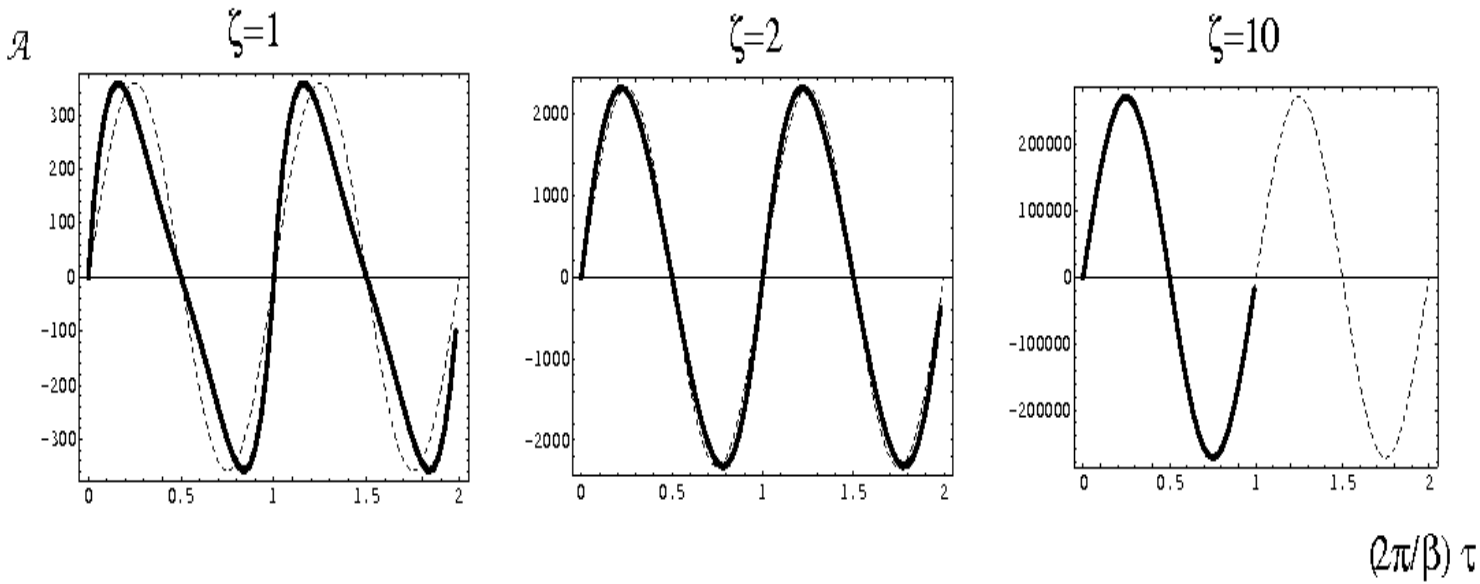

Figure 1. Saturation towards a harmonic Euclidean time dependence of the contributions of Harrington-Shepard calorons to the field-strength correlation defining the phase of the field $\phi$ as a function of the scaled cutoff $\xi \equiv \frac{\rho}{\beta}$ for the instanton-scale-parameter integration. Cutting off at $\rho \sim r_{0}=1.29 \beta_{0}$ suggests that there are (mild) deviations from a harmonic dependence. Figure taken from [25].

Still, the link between a hypothetically static monopole and its surroundings-described by infinite-volume thermodynamics - to the quantum-induced mass of the containing region due to oscillation is self-consistently made via Equation (5) by energy conservation, and reasonable estimates of $r_{0}$ and the critical temperature $T_{c}=13.87 /(2 \pi \times 118.6) m_{0}=$ $9.49 \mathrm{keV}$ [7] $\left(\lambda_{c} \equiv 2 \pi T_{c} / \Lambda=13.87\right.$ [1]) should yet be possible.

\section{Lowest Spherically Symmetric Breathing Mode}

Spherically symmetric oscillations of the deconfining plasma temperature $T_{0}$ are not only driven by internally induced monopole oscillations but interfere with spatially homogeneous, coherent plasma oscillations due to external distortions of the initial temperature: $T_{0} \longrightarrow T_{0}+\delta T$. At the same, radius $r_{0}$ of these two oscillation modes should be compared in terms of their frequencies $\omega_{0}$ and $\Omega_{0}$, respectively, in order to judge whether mass $m_{0}$ of the self-intersection region, arising from monopole-driven oscillation, is influenced sizably by the contribution of a global, oscillatory temperature distortion. Moreover, a neutral plasma ball of radius $R_{0} \gg r_{0}$ is subject to thermodynamics at face value. Since such a (distorted) macrocopic ball is expected to radiate electromagnetically on top of its weak black-body evaporation, diagnosing a lower frequency cutoff $\Omega_{0}$ in the excess spectrum would allow the unique extraction of plasma-ball radius $R_{0}$ and, hence, of the energy content of the ball. 
Let us, thus, compute the frequency $\Omega_{0}$ of the lowest spherically symmetric breathing mode of a deconfining SU(2) Yang-Mills plasma ball of homogeneous energy density $\rho$ and pressure $P$, for which its temperature oscillates about $T_{0}$. Surface effects, arising from the transition between the deconfining (bulk) and the confining (exterior of ball) phases can be neglected for a sufficiently large ball mass $M \equiv \frac{4}{3} \pi R_{0}^{3} \rho\left(R_{0}\right)$, and the expression for $\Omega_{0}$ reads as follows [26,27]:

$$
\Omega_{0}=\frac{\pi c_{s} \Lambda}{\bar{R}_{0}},
$$

where the dimensionless quantities $c_{s}$ (longitudinal sound velocity) and $\bar{R}_{0}\left(R_{0}\right.$ in units of the inverse Yang-Mills scale $\Lambda^{-1}$ ) are defined as follows:

$$
\left.c_{s}^{2} \equiv \frac{\frac{d \bar{P}}{d \lambda}}{\frac{d \bar{\rho}}{d \lambda}}\right|_{\lambda=\lambda_{0}}
$$

and $\bar{R}_{0} \equiv R_{0} \Lambda$. In Equation (12), the employments of the one-loop pressure $P \equiv \Lambda^{4} \bar{P}$ and of the one-loop energy density $\rho \equiv \Lambda^{4} \bar{\rho}$ are excellent approximations (modified by higher-loop corrections on the $1 \%$-level [1]). Amusingly, an estimate of $\Omega_{0}$ by virtue of a linearisation of the force-balance equation $\frac{4}{3} \pi R^{3} \rho(R) \ddot{R}=4 \pi R^{2} P(R)$ about the stable point $R_{0}$ and an appeal to energy conservation of the following:

$$
\bar{R} \equiv R \Lambda=\left(\frac{3 \bar{M}}{4 \pi \bar{\rho}(\bar{R})}\right)^{1 / 3} \quad(\bar{M} \equiv \Lambda M),
$$

replaces the factor of $\pi$ in Equation (11) by a factor of three. For $\bar{P}$ and $\bar{\rho}$, we have the following [1]:

$$
\begin{aligned}
\bar{P}(2 a, \lambda) & \equiv-\frac{2 \lambda^{4}}{(2 \pi)^{6}}[2 \tilde{P}(0)+6 \tilde{P}(2 a)]-2 \lambda, \\
\bar{\rho}(2 a, \lambda) & \equiv \frac{2 \lambda^{4}}{(2 \pi)^{6}}[2 \tilde{\rho}(0)+6 \tilde{\rho}(2 a)]+2 \lambda, \\
a & =a(\lambda) \equiv 2 \pi e(\lambda) \lambda^{-3 / 2} \quad\left(e\left(\lambda_{0}\right)=12.96\right),
\end{aligned}
$$

where

$$
\begin{aligned}
& \tilde{P}(y) \equiv \int_{0}^{\infty} d x x^{2} \log \left[1-\exp \left(-\sqrt{x^{2}+y^{2}}\right)\right], \\
& \tilde{\rho}(y) \equiv \int_{0}^{\infty} d x x^{2} \frac{\sqrt{x^{2}+y^{2}}}{\exp \left(\sqrt{x^{2}+y^{2}}\right)-1} .
\end{aligned}
$$

Taking into account implicit (via $a(\lambda)$ ) and explicit dependences of $\bar{P}$ and $\bar{\rho}$ on $\lambda$ and employing the evolution equation for the mass of off-Cartan gauge modes as a function of temperature [1]:

$$
1=-\frac{24 \lambda^{3}}{(2 \pi)^{6}}\left(\lambda \frac{d a}{d \lambda}+a\right) a D(2 a),
$$

one derives the following:

$$
\begin{aligned}
& \frac{d \bar{P}}{d \lambda}=-\frac{\lambda^{3}}{(2 \pi)^{6}}\left(16 \tilde{P}(0)+48\left(\tilde{P}(2 a)-a^{2} D(2 a)\right)\right), \\
& \frac{d \bar{\rho}}{d \lambda}=\frac{\lambda^{3}}{(2 \pi)^{6}}\left(16 \tilde{\rho}(0)+48\left(\tilde{\rho}(2 a)-a^{2}(D(2 a)-F(2 a))\right)\right)+2\left(1-\frac{D(2 a)-F(2 a)}{D(2 a)}\right),
\end{aligned}
$$


where the following is the case.

$$
\begin{aligned}
& D(y) \equiv \int_{0}^{\infty} d x \frac{x^{2}}{\sqrt{x^{2}+y^{2}}} \frac{1}{\exp \left(\sqrt{x^{2}+y^{2}}\right)-1} \\
& F(y) \equiv \int_{0}^{\infty} d x x^{2} \frac{\exp \left(\sqrt{x^{2}+y^{2}}\right)}{\left(\exp \left(\sqrt{x^{2}+y^{2}}\right)-1\right)^{2}}
\end{aligned}
$$

Substituting Equation (17) into Equation (12) at $\lambda_{0}=18.31$, we numerically obtain the following.

$$
c_{S}\left(\lambda_{0}\right)=0.479 .
$$

For Equation (11), this yields the following.

$$
\Omega_{0}=1.506 \frac{\Lambda}{\bar{R}_{0}} \text {. }
$$

Let us now compare the monopole-core induced frequency $\omega_{0}$ of the self-intersection region of the figure-eight shaped center-vortex loop (model of the electron) with $\Omega_{0}$ at one and the same radius:

$$
r_{0}=R_{0}=4.043 H_{\infty}^{-1}\left(T_{0}\right),
$$

see Equation (7). For $\Omega_{0}$, this yields the following:

$$
\Omega_{0}=0.372 H_{\infty}\left(T_{0}\right)
$$

such that the following is the case.

$$
\frac{\omega_{0}}{\Omega_{0}}=\frac{12.96}{0.372}=34.84
$$

Such a large ratio is natural since the oscillation in the self-intersection regionquantum initiated by caloron or anticaloron action-is induced by the classical dynamics of a monopole core $[4,5]$ for which its radius matches the reduced Compton wave length $l_{C}$, while the lowest symmetric breathing mode of the neutral deconfining ball is a consequence of longitudinal sound propagation in an approximate bulk thermodynamics. This bulk associates with $r_{0}$ being comparable to the Bohr radius [7].

Equation (20) is the more reliable the larger $\bar{R}_{0}$ is. Isotropy breaking effects, which associate with the neglected surface dynamics of the ball and/or the excitation of spherically non-symmetric oscillation states, cause this surface to (electromagnetically) radiate with a spectrum that is cut off towards the infrared at a frequency of about $v_{0} \sim \frac{\Omega_{0}}{2 \pi}$, corresponding to wave length $l_{0}=\frac{1}{v_{0}} \sim \frac{2 \pi R_{0}}{1.506}$.

\section{Summary and Discussion}

This paper's main purpose was to compare two situations in which a ball-like region of deconfining phase in SU(2) Yang-Mills thermodynamics oscillates about the zero of the pressure at temperature $T_{0}$ : (i) the charged self-intersection region of a figure-eight shaped, solitonic center-vortex loop (a model of the electron) containing an internally quantumperturbed BPS monopole, for which its classical core dynamics drives this oscillation of (circular) frequency $\omega_{0}$ (up to a factor $\hbar$ coincident with the rest energy $m_{0} c^{2}$ of the soliton [8,9]); and (ii) the homogeneous, neutral region being perturbed externally such that a lowest spherically symmetric oscillatory excitation of (circular) frequency $\Omega_{0}$ is excited thanks to a finite longitudinal speed of sound $c_{S}$. At the same radius, $r_{0}=R_{0}=\frac{4.043}{\pi T_{0}}$, we obtain $\frac{\omega_{0}}{\Omega_{0}}=34.84$. This hierarchy relates to the very different causes of oscillation in either case and assures that the right-hand side of the mass formula Equation (5) for an oscillation 
in the sense of (i) does not receive any sizable contributions from an oscillation in the sense of (ii).

Secondly, we have observed a numerical error in [7] concerning the estimate of the system radius $r_{0}$ in terms of the spatial coarse-graining scale $|\phi|^{-1}$ at $T_{0}$. The correct result states that finite-size corrections to infinite-volume thermodynamics cannot be excluded at face value since the region of self-intersection actually is contained deeply within the ball of spatial coarse-graining invoked in the derivation of the effective theory [1]. However, the asymptotic harmonic time dependence of the integrated field-strength correlation [1], required for the introduction of the field $\phi$, is approximately saturated when cutting the instanton-scale-parameter integration off at $r_{0}<|\phi|^{-1}$ already.

The present work only represents a first step in studying the plasma dynamics of a ball-like region of deconfining phase at $T_{0}$. More realistically, the physics of the boundary shell, repesenting the transitions from the deconfining via the preconfining to the confining phases, should be taken into account. Moreover, we did not address evaporation physics (in adiabatic approximation: emission of non-intersecting and self-intersecting centervortex loops and, assuming a mixing with an SU(2) Yang-Mills theory of much lower scale, electromagnetic modes from the surface of this shell in terms of thermal spectra) in the case of macroscopically sized balls (see [28]) and how this process affects oscillation dynamics. Last but not least, a thorough discussion of (delocalised) spin in terms of the center-flux along the figure-eight shaped vortex line and the emergence of the electric Coulomb field [29] throughout the confining-phase exterior to the blob of vortex-line selfintersection needs to be realised. The latter represents a small contribution to the soliton mass [7] and should manifest polarised dipole densities in (anti)caloron peripheries [1,30].

Our results on the spherically symmetric oscillations of the homogeneous and macroscopic plasma could be relevant in the description of certain, quasi-stabilised, compact and radiating objects created within atmospheric discharges and for plasma diagnostics in terrestial fusion experiments.

Author Contributions: The material of Sections 2 and 3 was originally conceived by R.H. Both authors, T.G. and R.H. have contributed equally to an intense discussion, correction, and conceptual consolidation of this first step. All authors have read and agreed to the published version of the manuscript.

Funding: This research received no external funding.

Institutional Review Board Statement: Not applicable.

Informed Consent Statement: Not applicable.

Data Availability Statement: Not applicable.

Acknowledgments: We would like to acknowledge a useful conversation with Anton Plech. Discussions with Manfried Faber on common themes and differences of his and the here-proposed soliton model are gratefully acknowledged.

Conflicts of Interest: The authors declare no conflict of interest.

\section{Notes}

For the sake of pointing out classical vs. quantum physics in association with (anti)calorons, we alternate between SI and natural units in this introduction, and from Section 2 onward, we exclusively work in natural units where the speed of light in vacuum, Planck's (reduced) quantum of action, and Boltzmann's constant are all set equal to unity: $c=\hbar=k_{B}=1$.

The radius $r_{0}$ is denoted $R_{0}$ in [7].

In physics models, charges and fluxes with respect to $\mathrm{U}(1) \subset \mathrm{SU}(2)$ need to be interpreted in an electric-magnetically dual manner [1]. 


\section{References}

1. Hofmann, R. The Thermodynamics of Quantum Yang-Mills Theory: Theory and Application; 2nd ed.; World Scientific: Singapore, 2016.

2. Del Debbio, L.; Manfried, F.; Greensite, J.; Olejnik, S. Center dominance, center vortices, and confinement. In Proceedings of the NATO Advanced Research Workshop on Theoretical Physics: New Developments in Quantum Field Theory, Zakopane, Poland, 14-20 June 1997; Volume 6, p. 47.

3. Diakonov, D.; Gromov, N.; Petrov, V.; Slizovskiy, S. Quantum weights of dyons and of instantons with nontrivial holonomy. Phys. Rev. D 2004, 70, 036003. [CrossRef]

4. Fodor, G.; Racz, I. What Does a Strongly Excited 't Hooft-Polyakov Magnetic Monopole Do? Phys. Rev. Lett. 2004, 92, 151801. [CrossRef]

5. Forgacs, P.; Volkov, M.S. Resonant Excitations of the 't Hooft-Polyakov Monopole. Phys. Rev. Lett. 2004, 92, 151802. [CrossRef]

6. Faber, M. A model for topological fermions. Few Body Syst. 2001, 30, 149. [CrossRef]

7. Hofmann, R. The isolated electron: De Broglie's "hidden" thermodynamics, SU(2) Quantum Yang-Mills theory, and a strongly perturbed BPS monopole. Entropy 2017, 19, 575. [CrossRef]

8. De Broglie, L. Recherches sur la Theorie Des Quanta. Annales de Physique 1925, 10 4925. [CrossRef]

9. De Broglie, L. The thermodynamics of the isolated particle. Great Probl. Sci. 1964, 17, 1.

10. Schrödinger, E. Quantisierung als Eigenwertproblem I. Ann. Phys. 1926, 79, 361. [CrossRef]

11. Schrödinger, E. Quantisierung als Eigenwertproblem II. Ann. Phys. 1926, 79, 489. [CrossRef]

12. Schrödinger, E. Quantisierung als Eigenwertproblem III. Ann. Phys. 1926, 80, 437. [CrossRef]

13. Schrödinger, E. Quantisierung als Eigenwertproblem IV. Ann. Phys. 1926, 81, 109. [CrossRef]

14. Born, M. Zur Quantenmechanik der Stoßvorgänge. Z. Phys. 1926, 37, 863. [CrossRef]

15. Moosmann, J.; Hofmann, R. Evolving center-vortex loops. ISRN Math. Phys. 2012, 2012, 236783. [CrossRef]

16. Nahm, W. A simple formalism for the BPS monopole. Phys. Lett. B 1980, 90, 413. [CrossRef]

17. Nahm, W. All self-dual multimonopoles for arbitrary gauge groups. In CERN Preprint TH-3172; CERN: Geneva, Switzerland, 1981.

18. Nahm, W. The Construction of all Self-dual Multimonopoles by the ADHM Method. In Monopoles in Quantum Field Theory; Craigie, N., Ed.; World Scientific: Singapore, 1982; p. 87.

19. Nahm, W. Self-dual monopoles and calorons. In Group Theoretical Methods in Physics; Springer: Berlin/Heidelberg, Germany, 1983; p. 189.

20. Kraan, T.C.; Van Baal, P. Exact, T-duality, between calorons, Taub-NUTspaces. Phys. Lett. B 1998, 428, 268. [CrossRef]

21. Kraan, T.C.; Van Baal, P. Periodic instantons with non-trivial holonomy. Nucl. Phys. B 1998, 533, 627. [CrossRef]

22. Lee, K.; Lu, C. SU(2) calorons and magnetic monopoles. Phys. Rev. D 1998, 58, 025011. [CrossRef]

23. 't Hooft, G. On the phase transition towards permanent quark confinement. Nucl. Phys. B 1978, 138, 1. [CrossRef]

24. Prasad, M.K.; Sommerfield, C.M. Exact Classical Solution for the 't Hooft Monopole and the Julia-Zee Dyon. Phys. Rev. Lett. 1975, 35, 760. [CrossRef]

25. Herbst, U.; Hofmann, R. Emergent Inert Adjoint Scalar Field in SU(2) Yang-Mills Thermodynamics due to Coarse-Grained Topological Fluctuations. ISRN High Energy Phys. 2012, 2012, 373121. [CrossRef]

26. Hartl, G.V.; Hu, M.; Sader, J.E. Softening of the symmetry breaking mode in gold particles by laser-induced heating. J. Phys. Chem. B 2003, 107, 7472. [CrossRef]

27. Lamb, H. On the vibrations of an elastic sphere. Proc. Lond. Math. Soc. 1882, 13, 189. [CrossRef]

28. Giacosa, F.; Hofmann, R. Bursts of low-energy electron-positron pairs in TeV-range collider physics. Mod. Phys. Lett. A 2009, 24, 1937. [CrossRef]

29. Faber, M. A Geometric Model in 3+1D Space-Time for Electrodynamical Phenomena. Universe 2022, 8, 73. [CrossRef]

30. Grandou, T.; Hofmann, R. Thermal ground state and nonthermal probes. Adv. Math. Phys. 2015, 2015, 197197. [CrossRef] 